\title{
A DERIVATIVE IN THE SETTING OF OPERATOR-VALUED MEASURES
}

\author{
J. R. EDWARDS AND S. G. WAYMENT
}

\begin{abstract}
There has been considerable recent development concerning integration of vector-valued functions with respect to operator-valued measures, including various Radon-Nikodym type theorems. This paper is concerned with obtaining an analytic definition of derivative which will act as an inverse operator for these integration processes. A derivative is defined and, with additional conditions imposed on the measure, the two fundamental theorems of calculus are proved, i.e. that differentiation and integration are indeed inverse processes. Several examples are discussed to point out the shortcomings of this definition of derivative. Since this paper was written, some partial work has been done by D. H. Tucker and S. G. Wayment to adjust the definition to remove some of these defects. However, they were not then able to establish certain desirable theorems. For further details, the reader is referred to the Proc. Sympos. on Vector and Operator Valued Measures and Applications (Snowbird, Utah, 1972).
\end{abstract}

1. Introduction. To date there has been a considerable effort expended toward generalizing the notion of integration into abstract settings. Part of this effort is found in [2], [3], [4], [7], and [8]. A question which arises naturally from these efforts is: Does there exist a notion of derivative which parallels such developments? A partial answer to this question was evolved by S. G. Wayment in his doctoral dissertation [9] where he defined conditions under which a "Radon-Nikodym" theorem is valid. D. H. Tucker raised the following question in a private communication: Is there an analytic definition for the derivative in the setting of operator measures which matches this Radon-Nikodym derivative?

The subject of this paper is to give an affirmative answer to part of the question; two definitions of derivative are given, one of which we call the weak derivative and the other the strong derivative. In order to establish these definitions as acceptable extensions of the usual derivative

Received by the editors January 19, 1972 and, in revised form, August 22, 1972.

AMS (MOS) subject classifications (1969). Primary 2816, 2850.

Key words and phrases. Linear topological, Hausdorff space, locally compact Hausdorff space, bounded semivariation, weakly differentiable, strongly differentiable, locally nondispersive, Fundamental Theorem of Calculus. 
we establish some of the fundamental rules for differentiation, and in the case of the strong derivative we establish that if the derivative of a set function is continuous, then the set function is retrieved by integrating the derivative, which is essentially half of the Fundamental Theorem of Calculus. By placing additional requirements on the underlying measure of integration we establish the other half of the Fundamental Theorem, i.e. that the derivative of a set function which is defined as the integral of a continuous function is that continuous function.

2. The general setting and basic definitions. For the purpose of this paper, we suppose that $X$ and $Y$ are both linear topological Hausdorff spaces, and that $L[X, Y]$ is the set of continuous linear operators from $X$ into $Y$. Let $H$ denote a locally compact Hausdorff space and let $\Sigma$ denote the ring of sets generated by the compact subsets of $H$. Also, $K$ shall denote a finitely additive set function on $\Sigma$ with values in $L[X, Y]$, which is of bounded semivariation ([3] or [8]), i.e., $K$ is said to be of bounded semivariation if given a neighborhood $U$ of $\theta_{Y}$, the origin of $Y$, there is a neighborhood $V$ of $\theta_{X}$, the origin of $X$, such that if $\left\{e_{i}\right\}_{i=1}^{n}$ is a partition of $H$ over $\Sigma$ and $x_{i} \in V, i=1, \cdots, n$, then $\Sigma\left[K\left(e_{i}\right)\right]\left(x_{i}\right) \in U$.

The following definition defines what is meant by weak derivative. For the purposes of the definition $G$ denotes a finitely additive set function from $\Sigma$ with values in $Y$.

2.1. Definition. The set function $G$ is said to be weakly differentiable with respect to $K$ at the point $t \in H$ providing $\bigcap_{e}\left\{\bigcup_{e^{\prime}}\left\{\left[K\left(e^{\prime}\right)\right]^{-1} G\left(e^{\prime}\right): e^{\prime} \subset e\right.\right.$ is a neighborhood of $t\}: e$ is a neighborhood of $t\}$ is a singleton. If the derivative exists it shall be denoted by $(d G / d K)(t)$.

In [7] and [9], $G$ is defined to be absolutely continuous with respect to $K$ provided that for each $E$ in $\Sigma$ we have $G(E) \in\left\{\bigcup_{\sigma}\left\{\sum_{\sigma} K\left(e_{i}\right) \cdot X\right\}: \sigma\right.$ a partition of $E\}$. The similarities are apparent.

It is a straightforward argument to establish that if $X, Y$, and $H$ are each the reals, then this is the usual notion of derivative provided the closure is taken in the extended reals, i.e., $+\infty$ and $-\infty$ are allowed in $Y$ (see Example 2.4). Because of this it is not surprising that the following remark is true.

2.2. REMARK. The derivative is a linear operator on the space of weakly differentiable finitely additive set functions which have $\Sigma$ as their domain and which take values in $Y$.

The proof of the remark follows directly from observing that if $G$ and $F$ are two such set functions, then for each $e \in \Sigma$,

$$
[K(e)]^{-1}(\alpha G(e)+\beta F(e))=\alpha[K(e)]^{-1}(G(e))+\beta[K(e)]^{-1}(F(e))
$$

for scalars $\alpha$ and $\beta$. 
In general, the above definition of derivative at a point $t$ does not require that as $e$ "squeezes" down on the point $t$ that $\left[K\left(e^{\prime}\right)\right]^{-1}\left(G\left(e^{\prime}\right)\right)$ have any points at all close to $(d G / d K)(t)$ for all $e^{\prime}$. In the classical setting this property results from the definition. This property of "squeezing" appears to be critical to many of the theorems which deal with derivatives and it is this thought which motivates the following definition of strong derivative. We assume that the set function $G$ is defined as before.

2.3. Definition. The set function $G$ is said to be strongly differentiable with respect to $K$ at the point $t \in H$ providing:

(1) $G$ is weakly differentiable at $t$.

(2) Given a neighborhood $U$ of $(d G / d K)(t)$ there is a neighborhood $e$ of $t$ such that if $e^{\prime} \subset e$ is a neighborhood of $t$ then $\left\{\left[K\left(e^{\prime}\right)\right]^{-1}\left(G\left(e^{\prime}\right)\right)\right\} \cap U \neq \varnothing$.

If the strong derivative exists at $t$ it shall be denoted by $D_{K} G(t)$. We note that in the real setting it is not necessary to consider extended real numbers for $Y$ when establishing the equivalence of the ordinary derivative with the strong derivative.

EXAMPLE 2.4. Let $G_{1}(x)$ be the real-valued function on $[0,1]$ which is 1 at each rational and 0 at each irrational and let $G_{2}(x)$ be $x$ at each rational and 0 at each irrational. Let $K$ be ordinary length defined on half open intervals and closed intervals containing 0 . Then $G_{1}$ is weakly differentiable if the closure is not taken in the extended reals, while $G_{2}$ is not weakly differentiable since

$$
\bigcap_{e}\left\{\bigcup_{e^{\prime}}\left\{\left[K\left(e^{\prime}\right)\right]^{-1} G\left(e^{\prime}\right)\right\}\right\}=\{0,1\} .
$$

It is this behavior in $G_{1}$ that motivates us to include (2) of Definition 2.3. That is, we do not want to allow $d G / d K$ to exist at $t$ when $\left[K\left(e^{\prime}\right)\right]^{-1} G\left(e^{\prime}\right)$ sometimes contains points near some vector $x_{0}$ and also sometimes contains arbitrarily large vectors which are all eliminated in the intersection process.

REMARK 2.5. One might be tempted to replace condition (2) of Definition 2.3 by the seemingly more natural condition $\left[K\left(e^{\prime}\right)\right]^{-1} G\left(e^{\prime}\right) \subset U$. However, recall that $K\left(e^{\prime}\right)$ is an operator from $X$ to $Y$ and if the kernel is nontrivial, then an entire hyperplane is contained in $\left[K\left(e^{\prime}\right)\right]^{-1} G\left(e^{\prime}\right)$. Thus only in the case of very special topologies on $X$ could one expect containment. In view of Example 2.4 it becomes somewhat surprising that Definition 2.3 is sufficient.

3. The Fundamental Theorem. It is with Definition 2.3 that we are able to establish the "Fundamental Theorem of Calculus" in our stated setting.

3.1. THEOREM. If $f(t)=D_{K^{\prime}} G(t)$ is a continuous function on $H$, then for each $E \in \Sigma, \int_{E} d K \cdot f=G(E)$. 
Proof. First the reader is asked to observe that it follows from results in [3] that the integral exists over each $E \in \Sigma$. The general plan of attack in establishing this result is to show given a neighborhood of $U$ of $\theta_{Y}$ that $\int_{E} d K \cdot f-G(E) \in U$. Our first step in establishing this result is to observe that since $K$ is of bounded semivariation there is a neighborhood $V$ of $\theta_{X}$ such that if $\left\{x_{i}\right\}_{i=1}^{n} \subset V, \Sigma\left[K\left(e_{i}\right)\right]\left(x_{i}\right) \in U^{\prime}$ for every ring partition $\left\{e_{i}\right\}_{i=1}^{n}$ of $H$ over $\Sigma$, where $U^{\prime}$ is a neighborhood of $\theta_{Y}$ such that $U^{\prime}+U^{\prime}+$ $U^{\prime} \subset U$. Further observe that $E \in \Sigma$ implies that $\bar{E}$ is compact. Since $f(t)$ is a strong derivative for every point $t \in H$, it follows that for each $t \in \bar{E}$ there is a neighborhood $e_{t}$ of $t$ such if $e_{t}^{\prime}$ is a neighborhood of $t$ contained in $e_{t}$ there is an $x_{t}^{\prime}$ contained in $\left\{\left[K\left(e_{t}^{\prime}\right)\right]^{-1}\left(G\left(e_{t}^{\prime}\right)\right)\right\} \cap\left(V+D_{K} G(t)\right)$. Furthermore, since $f$ is continuous we may assume that each $e_{t}$ is chosen such that $t^{\prime} \in e_{t}$ implies $f(t)-f\left(t^{\prime}\right) \in V$. The compactness of $\bar{E}$ implies that there is a finite subcover $\left\{e_{t_{i}}\right\}_{i=1}^{n}$ of $\left\{e_{t}: t \in \bar{E}\right\}$ which covers $\bar{E}$. Since $f$ is integrable with respect to $K$ over $E$, it follows that there is a partition $\left\{e_{i}\right\}_{i=1}^{m}=\Pi$ which refines the partition of $E$ defined by $\left\{e_{t_{i}} \cap E: i=1, \cdots, n\right\}$ such that

$$
\int_{E} d K \cdot f-\Sigma_{\Pi}\left[K\left(e_{i}\right)\right]\left(f\left(h_{i}\right)\right) \in U^{\prime}
$$

where $h_{i} \in e_{i}$ for $i=1,2, \cdots, m$. The choice of $\Pi$ implies that for each $i$ there is a $j(i)$ such that $e_{i} \subset e_{t_{j(i)}}$ from which we conclude that $f\left(t_{j(i)}\right)$ $f\left(h_{i}\right) \in V$ for each $i$. In order to introduce notation which will simplify a later step we observe that for each $i,\left[K\left(e_{i}\right)\right]\left\{\left[K\left(e_{i}\right)\right]^{-1}\left(G\left(e_{i}\right)\right)\right\}$ is the single point $G\left(e_{i}\right)$; hence it follows for each $i$ and for any $x \in\left[K\left(e_{i}\right)\right]^{-1}\left(G\left(e_{i}\right)\right)$ that $\left[K\left(e_{i}\right)\right](x)=\left[K\left(e_{i}\right)\right]\left\{\left[K\left(e_{i}\right)\right]^{-1}\left(G\left(e_{i}\right)\right)\right\}$. For each $i$, let $x_{i}$ denote the element of $\left\{\left[K\left(e_{i}\right)\right]^{-1}\left(G\left(e_{i}\right)\right)\right\} \cap\left\{V+f\left(t_{j(i)}\right)\right\}$ which is guaranteed because $f(t)$ is a strong derivative. We are now in a position to finalize our argument. Observe that

$$
\begin{aligned}
\int_{E} d K & \cdot f-G(E)=\int_{E} d K \cdot f-\Sigma\left[K\left(e_{i}\right)\right]\left\{\left[K\left(e_{i}\right)\right]^{-1}\left(G\left(e_{i}\right)\right)\right\} \\
= & \left(\int_{E} d K \cdot f-\Sigma_{\Pi}\left[K\left(e_{i}\right)\right]\left(f\left(h_{i}\right)\right)\right)+\left(\Sigma_{\Pi}\left[K\left(e_{i}\right)\right]\left(f\left(h_{i}\right)\right)-\Sigma_{\Pi}\left[K\left(e_{i}\right)\right]\left(x_{i}\right)\right) \\
= & \left(\int_{E} d K \cdot f-\Sigma_{\Pi}\left[K\left(e_{i}\right)\right]\left(f\left(h_{i}\right)\right)\right) \\
& +\left(\Sigma_{\Pi}\left[K\left(e_{i}\right)\right]\left(f\left(h_{i}\right)\right)-\Sigma_{\Pi}\left[K\left(e_{i}\right)\right]\left(f\left(t_{j(i)}\right)\right)\right) \\
& +\left(\Sigma\left[K\left(e_{i}\right)\right]\left(f\left(t_{j(i)}\right)\right)-\Sigma\left[K\left(e_{i}\right)\right]\left(x_{i}\right)\right) \\
= & \left(\int_{E} d K \cdot f-\Sigma_{\Pi}\left[K\left(e_{i}\right)\right]\left(f\left(h_{i}\right)\right)\right)+\left(\Sigma_{\Pi}\left[K\left(e_{i}\right)\right]\left(f\left(h_{i}\right)-f\left(t_{j(i)}\right)\right)\right) \\
& +\left(\Sigma\left[K\left(e_{i}\right)\right]\left(f\left(t_{j(i)}\right)-x_{i}\right)\right) \\
\in & U^{\prime}+U^{\prime}+U^{\prime} .
\end{aligned}
$$


In the first case the inclusion follows from the choice of the partition and in the other two cases the inclusion follows from the fact that $K$ is of bounded semivariation and for each $i, f\left(h_{i}\right)-f\left(t_{j(i)}\right)$ and $f\left(t_{j(i)}\right)-x_{i}$ are elements of $V$. From the choice of $U^{\prime}$ it follows that

$$
\int_{E} d K \cdot f-G(E) \in U
$$

and the theorem is proved.

In order to establish the converse of this theorem, and thereby demonstrate that strongly differentiable set functions exist, we need to provide a further requirement on the underlying measure of integration. For this purpose the following definition is made.

3.2. Definition. A set function $K$ is said to be locally nondispersive at the point $t \in H$ providing there is an $E \in \Sigma$ which is a neighborhood of $t$ such that:

(1) For each $e \subset E$ which is in $\Sigma, K(e)$ is $1-1$ and $[K(e)]^{-1}$ is continuous on $[K(e)](X)$.

(2) There is a scalar $B$ such that if $\left\{x_{i}\right\} \subset U$, a neighborhood of $\theta_{X}$, and $e \subset E$ is in $\Sigma$, then $[K(e)]^{-1}\left(\Sigma\left[K\left(e_{i}\right)\right]\left(x_{i}\right)\right) \in B U$ for each partition $\left\{e_{i}\right\}$ of $e$.

In the setting where $X$ and $Y$ are linear normed spaces a property which implies that $K$ is locally nondispersive is that $K(e)$ is 1 to 1 and $[K(e)]^{-1}$ is continuous on $[K(e)](X)$ for each $e$, and that there is a constant $B$ such that $\|K(e)\| \geqq B W K_{e}$, where $W K_{e}$ is the semivariation of $K$ over $e$.

The converse of Theorem 3.1 is now given.

3.3. THEOREM. Suppose $f$ is a continuous function from $H$ into $X$ and that $K$ is locally nondispersive. Then if the set function $G$ from $\Sigma$ to $Y$ defined by $G(e)=\int_{r} d K \cdot f, e \in \Sigma$, satisfies $G(e) \in[K(e)](X)$, for each $e \in \Sigma$, it is strongly differentiable. Furthermore, $D_{K} G(t)=f(t)$ for each $t \in H$.

Proof. In order to establish the result we show that given $t \in H$ and a neighborhood $U$ of $0_{X}$ there is a neighborhood $e$ of $t$ such that $\bigcup\left\{\left[K\left(e^{\prime}\right)\right]^{-1}\left(G\left(e^{\prime}\right)\right): e^{\prime}\right.$ is a neighborhood of $t$ and $\left.e^{\prime} \subset e\right\} \subset U+f(t)$. From this it will follow immediately that $\bigcap_{0}\left\{\bigcup_{e^{\prime}}\left\{\left[K\left(e^{\prime}\right)\right]^{-1}\left(G\left(e^{\prime}\right)\right): e^{\prime}\right.\right.$ is a neighborhood of $t$ and $\left.e^{\prime} \subset e\right\}: e$ is a neighborhood of $\left.t\right\}=f(t)$ which implies that $G$ is weakly differentiable. That $G$ is strongly differentiable is clear because $[K(e)]^{-1}$ is $1-1$. We proceed to show the desired containment. Choose the neighborhood $U^{\prime}$ of $\theta_{X}$ such that $U^{\prime}+U^{\prime} \subset U$. The continuity of $f$ guarantees there is a neighborhood $e$ of $t$ such that for $t^{\prime} \in e, f(t)-$ $f\left(t^{\prime}\right) \in U^{\prime} \mid B$ where $B$ is the constant assured by the fact that $K$ is locally nondispersive. Since $[K(e)]^{-1}$ on $[K(e)](X)$ is continuous, there is a neighborhood $V$ of $\theta_{Y}$ such that $[K(e)]^{-1}(V) \subset U^{\prime}$. 
Suppose $e^{\prime} \subset e$ is a neighborhood of $t$. Let $x=\left[K\left(e^{\prime}\right)\right]^{-1}(G(e))$. Since $G\left(e^{\prime}\right)=\int_{e^{\prime}} d K \cdot f$, there is a partition $\left\{e_{i}\right\}$ of $e^{\prime}$ over $\Sigma$ such that

$$
G\left(e^{\prime}\right)-\Sigma\left[K\left(e_{i}\right)\right] f\left(h_{i}\right) \in V,
$$

where $h_{i} \in e_{i}$ for each $i$; hence, the continuity of $\left[K\left(e^{\prime}\right)\right]^{-1}$ implies that $x-\left[K\left(e^{\prime}\right)\right]^{-1}\left(\sum K\left(e_{i}\right)\left(f\left(h_{i}\right)\right)\right) \in U^{\prime}$ where $x=\left[K\left(e^{\prime}\right)\right]^{-1}\left(G\left(e^{\prime}\right)\right)$ for each $i, x_{i}$ shall denote $f(t)-f\left(h_{i}\right)$. The choice of $e^{\prime}$ insures that $x_{i} \in U^{\prime} \mid B$. Therefore

which implies

$$
x-\left[K\left(e^{\prime}\right)\right]^{-1}\left(\Sigma\left[K\left(e_{i}\right)\right]\left(f(t)+x_{i}\right)\right) \in U^{\prime}
$$

$$
x-f(t)-\left[K\left(e^{\prime}\right)\right]^{-1}\left(\Sigma\left[K\left(e_{i}\right)\right]\left(x_{i}\right)\right) \in U^{\prime}
$$

or written differently

$$
x-f(t) \in U^{\prime}+\left\{\left[K\left(e^{\prime}\right)\right]^{-1}\left(\Sigma\left[K\left(e_{i}\right)\right]\left(x_{i}\right)\right)\right\} .
$$

Since $K$ is locally nondispersive with associated scalar $B$, the fact that $x_{i} \in U / B$ for each $i$ implies that $\left[K\left(e^{\prime}\right)\right]^{-1}\left(\Sigma\left[K\left(e_{i}\right)\right]\left(x_{i}\right)\right) \in U^{\prime}$; and, therefore we conclude that

$$
x-f(t) \in U^{\prime}+U^{\prime} \subset U
$$

which implies that for each neighborhood $e^{\prime}$ of $t$ which is contained in $e$,

This implies

$$
\left[K\left(e^{\prime}\right)\right]^{-1}\left(G\left(e^{\prime}\right)\right) \in U+f(t) .
$$

$\bigcup_{e^{\prime}}\left\{\left[K\left(e^{\prime}\right)\right]^{-1}\left(G\left(e^{\prime}\right)\right): e^{\prime}\right.$ is a neighborhood of $t$ and $\left.e^{\prime} \subset e\right\} \subset U+f(t)$

and the theorem is established.

4. Concluding remarks. Definitions 2.1 and 2.2 can be easily generalized in the following manner. Let $M$ be a set in an arbitrary topological space $H$, and let $G$ be a function defined on a certain class of sets in $H$ (which includes $M$ ) into subsets of a space $Y$. Suppose $e$ and $e^{\prime}$ are from a field of sets (perhaps neighborhoods) containing $M$ such that $e^{\prime} \subset e$, and suppose for each set $e, K(e)$ is a mapping from some space $X$ into $Y$. Then Definition 2.1 can be applied to define a generalized derivative whether or not it yields a singleton. If a singleton is produced it could be called the weak generalized derivative. It is not known if such a definition would prove useful. However, the authors believe significant gains could be made in the above theory by reformulating integration and differentiation to include set-valued set functions.

The additional conditions on the measure for Theorem 3.3 over 3.1 are somewhat unsatisfactory and we next give an example to show they are necessary for the proof as given and some definitions for the purpose of stating a conjecture concerning a relaxation of those conditions. 
4.1. Example. Let $H=[0,1]$ and let $\Sigma=\{$ the set of all half open intervals $(a, b]$ and closed intervals $[0, a]\}$. Let $X=R$, the real numbers and let $Y=C[0,1]=\{$ the set of functions from $H$ into $X\}$. Tucker [6] has used the transformation $T(f)=f \in Y$ to construct a measure $K$ for other purposes which in some sense might be considered as the "canonical bad example". Indeed this measure appears to have all of the pathological behavior that distinguishes real countably additive measures from operator valued finitely additive measures. If $E \in \Sigma$ then $K(E)$ is the characteristic function of $E$ and maps $X=R$ into $Y^{+}$(the weak sequential extension of $Y$ in $Y^{* *}$ ) via $K(E): \alpha \rightarrow \alpha K(E)=\alpha \chi_{E}$. Let $f(t)=t$ and let $G(E)=$ $\int_{E} d K \cdot f=f \chi_{E}$. Then $G(E)$ is not in the range of $K(E)$ for any $E$. Indeed, $K$ has only scalar multiples of the characteristic function of $E$ in its range, and hence cannot operate on $G(E)$. It is also easily seen that similarly condition (2) of the Definition 3.2 of nondispersive fails to hold. Hence even though $G$ arises from an integration, the derivative as defined herein cannot exist. This defect is removed in the paper referred to in the abstract.

4.2. Definition. The set function $K$ is said to be weakly, locally nondispersive at $t \in H$ providing that given a neighborhood of $U$ of $\theta_{X}$ there is a neighborhood $e$ of $t$ which is in $\Sigma$ and a neighborhood $V$ of $\theta_{X}$ such that if $\left\{e_{i}\right\}$ is a partition of $e$ over $\Sigma$ and if $x_{i} \in V$ for each $i$, then $\left\{[K(e)]^{-1}\left(\Sigma\left[K\left(e_{i}\right)\right]\left(x_{i}\right)\right)\right\} \cap U \neq \varnothing$.

We next state our conjecture.

4.3. Conjecture. If in Theorem 3.3 the condition on $K$ that it be locally nondispersive at each $t \in H$ is replaced with the following conditions, then the results of the theorem still hold.

(1) $K$ is weakly, locally nondispersive at each $t \in H$.

(2) $\bigcap_{e}\left\{[K(e)]^{-1}([K(e)](f(t))): e \in \Sigma\right.$ is a neighborhood of $\left.t\right\}=f(t)$ for each $t \in H$.

(3) $\bigcap_{U}\left\{\bigcap_{e}\left\{[K(e)]^{-1}\left(\Sigma\left[K\left(e_{i}\right)\right]\left(x_{i}\right)\right):\left\{e_{i}\right\}\right.\right.$ is a partition of $e \in \Sigma$, a neighborhood of $\left.t ;\left\{x_{i}\right\} \subset U\right\}: U$ is a neighborhood of $\left.\theta_{X}\right\}=\varnothing$.

We conclude this paper with the following two questions and some partial results on one of them. In the Radon-Nikodym theorem stated in [5] it is proved that if the set function $G$ is absolutely continuous with respect to the set function $K$ there is a "blur" $\beta$ such that $G(E)=\int_{E} d K \cdot \beta$.

4.4. Question. If the blur $\beta$ identified by the referenced theorem is a function, is $\beta$ the weak derivative of $G$ with respect to $K$ ?

4.5. Question. In Theorem 3.3, the condition that $G(e) \subset[K(e)](X)$ for each $e$ is rather strong and not satisfied for certain well-known pairs $G$ and $K$ arising from integrals (see Example 4.1). Can this condition be weakened, possibly by defining a more complicated derivative?

In considering Question 4.4, the following example is enlightening. Let $H=[0,1]$ and let $X=E^{2}$, the euclidean plane. Let $\Sigma$ be as in Example 
4.1 and let $K(E)$ be the projection operator to the $y$-axis if $\frac{1}{2} \in E$ and $K(E)$ be the zero operator otherwise.

Finally let $f$ be any continuous function so that $f\left(\frac{1}{2}\right)=\left(\frac{1}{3}, 1\right)$. Then $\int_{E} d K \cdot f=(0,1)$ if $\frac{1}{2} \in E$ and zero otherwise. Any continuous function $f: E^{1}=$ $R \rightarrow E^{2}$ satisfying $f\left(\frac{1}{2}\right)=(t, 1)$ for any value of $t$ will serve as a function for the Radon-Nikodym theorem referenced in 4.4. The derivative as defined in this paper fails to exist since one does not obtain a singleton but rather the hyperplane of all points of the form $(t, 1)$.

A partial connection can be made however. In [5] Maynard gives a Radon-Nikodym theorem which retrieves a function instead of a blur for operator valued measures under the more restrictive conditions that

(1) $K$ and $m$ be of finite total variation (as opposed to bounded semivariation),

(2) $m \ll K$ in the zero implies zero sense, and

(3) for each nonnull $E$ there exists an $F \subset E$ and a compact set $W \subset X$ such that $F$ is localized in $W$ (see [5]).

If we assume the conditions of Maynard and the conditions of Theorem 3.3, then the function given by Maynard and our derivative function are the same since

$$
\left[K\left(e^{\prime}\right)\right]^{-1} G\left(e^{\prime}\right)=\left[K\left(e^{\prime}\right)\right]^{-1}\left(\Sigma G\left(e_{i}\right)\right)=\left[K\left(e^{\prime}\right)\right]^{-1}\left(\Sigma\left[x_{0} K\left(e_{i}\right)+\eta_{i}\right]\right)
$$

where $x_{0}$ is a fixed vector in $X$ and $\left\|\eta_{i}\right\| \leqq \varepsilon|K|_{e_{i}}$. Then

$$
\left[K\left(e^{\prime}\right)\right]^{-1} G\left(e^{\prime}\right)=x_{0}+K\left(e^{\prime}\right)^{-1} \Sigma \eta_{i} .
$$

But $\left\|\Sigma \eta_{i}\right\|<\varepsilon|K|_{e^{\prime}}$ and so continuity of $K^{-1}$ guarantees $\left[K\left(e^{\prime}\right)\right]^{-1}\left(\Sigma \eta_{i}\right)$ is small and $d G / d K=f(t)$ where $f$ is the function guaranteed by the RadonNikodym theorem in [5].

\section{REFERENCES}

1. N. Dunford and J. T. Schwartz, Linear operators I: General theory, Pure and Appl. Math., vol. 7, Interscience, New York, 1958. MR 22 \#8302.

2. R. J. Easton and D. H. Tucker, A generalized Lebesgue-type integral, Math. Ann. 181 (1969), 311-324.

3. J. R. Edwards and S. G. Wayment, Integral representations for continuous linear operators in the setting of convex topological vector spaces, Trans. Amer. Math. Soc. 157 (1971), 329-345. MR 43 \#7581.

4. R. K. Goodrich, A Riesz representation theorem in the setting of locally convex spaces, Trans. Amer. Math. Soc. 131 (1968), 246-258. MR 36 \#5731.

5. H. B. Maynard, A Radon-Nikodym theorem for finitely additive bounded measures, Pacific J. Math. (to appear).

6. D. H. Tucker, A representation theorem for a continuous linear transformation on a space of continuous functions, Proc. Amer. Math. Soc. 16 (1965), 946-953. MR 33 \#7865.

7. D. H. Tucker and S. G. Wayment, Absolute continuity and the Radon-Nikodym theorem, J. Reine Angew. Math. 244 (1970), 1-19. MR 42 \#7859. 
1973] DERIVATIVE IN THE SETTING OF OPERATOR-VALUED MEASURES 531

8. A. H. Shuchat, Integral representation theorems in topological vector spaces, Trans. Amer. Math. Soc. 172 (1972), 373-397.

9. S. G. Wayment, Absolute continuity and the Radon theorem, Ph.D. Thesis, University of Utah, Salt Lake City, Utah, 1968.

Department of Mathematics, Utah State University, Logan, Utah 84321

Current address (J. R. Edwards): U.S. Army, Frankfurt, Federal Republic of Germany

Current address (S. G. Wayment): University of Texas at San Antonio, San Antonio, Texas 78228 